\title{
Immuno-alignment Effect due to Mass DIV Immunization in Rapidly Evolving Viral Environments
}

\author{
Serge Dolgikh [0000-0001-5929-8954] \\ Dept. of Information Technology, \\ National Aviation University
}

\begin{abstract}
Direct to immune systems vaccines (DIV, such as mRNA) offered hope and promise in prevention of a number of conditions, including infectious viral disease. A strong advantage of this method is the ability to influence immune response reliably and predictably in the general population. While the promise and advantages of the approach are undeniable, we argue that in the plane of mass immunization of general population, it needs to be applied with caution specifically, with consideration of possible longer-term effects to prevent and avoid unintended consequences. One such possibility that is considered in this work is the effect of alignment or polarization of population immunity that can cause under certain conditions, as a side effect, accelerated production of resistant strains.
\end{abstract}

Keywords: Infectious epidemiology; mathematical modeling; immunity; mass immunization, direct-to-immune system vaccines.

\section{Introduction}

Direct to immune systems vaccines (such as mRNA) offered hope and promise in prevention of a number of conditions, including infectious viral dis-ease. A strong advantage distinct feature of this novel direction in vaccinology is the ability to influence immune response reliably and predictably in general population directly via manipulation of cell protein production mechanisms [1], as opposed to exposing the immune system to natural infectious agents in some form, in conventional immunization.

Over the recent years the methods were proven feasible and effective, including mass production of Covid-19 vaccines [2].

The intent of this work is to investigate scenarios and effects of prolonged exposure of the general population to direct to immune system (DIV) vaccines, for example, in the context of mass immunization. Analysis of interactions of virus with immunity system of the population with methods of mathematical modeling is applied to identify and discuss possible scenarios of interaction and development of an infectious variants or strains, over time. Of specific interest in this study are rapidly evolving viral agents with ability to mutate quickly and unpredictably, represented by well known examples 
of viral infections and longer-term effects of their interaction with population that was immunized on a significant (mass) scale.

\section{Methodology}

An analysis of interactions of an infectious agent with the population immunity is performed on different scales modeled by exposure clusters. Factors such as immunity variation in the population at different scales (represented by exposure clusters of different size) and mutative ability of the infection agent contribute to produce different scenarios of development of an infectious epidemics in the population.

\subsection{Population Immunity}

It will be assumed that in the general population, individual immune response to an infectious agent $G$, defined by the capacity to activate counter-agent mechanisms; while avoiding severe and harmful self-immune reactions is described by a probability distribution $X\left(p_{l}, . . p_{N}\right)$ defined by the immunity distribution parameters $P=\left\{p_{k}\right\}$.

The result of an exposure of an individual to a specific infectious agent $G$ can then described by the immunity response function: $\varphi(p, g)$ where $p$, the immunity parameters; $g$, the parameters describing infectious agent.

An essential observation in this framework of analysis is that in a group of individuals, certain variation of immunity parameters will be observed so the result of an exposure may depend, and possibly to a significant extent, on the composition of the group.

\subsection{Exposure Clusters}

Within the formulated framework and definitions, one can consider different groups in the population and analyze possible scenarios of development of the epidemics caused by infectious agent based on 1) the composition of the population groups, that can be defined by further parameters such as size; context (family; work; interests) and other relevant factors; 2) variation of the immunity parameters $P$ in the group; and 3 ) the parameters of the infectious agent, $G=\left\{g_{i}\right\}$.

For example, in a smallest cluster group (Level 0 cluster) such as conventional family group, immunity factor set $P$ is defined by two, possibly essentially different, sets of immunity parameters of parents $\left(P_{a}, P_{b}\right)$ and their combinations defined by genetic mechanisms. Therefore, the outcome of encountering the agent by two different families can be different, depending on variation of immunity factors describing resistance to the agent in the group as shown in Table 1.

Based on this observation, population clusters exposed to an infectious agent can be differentiated by their attitude, or resistance to the agent $G$ on a scale from strongly resistant (type VS, Table 1) to conductive (type W). In fact, the attitude has to be relative to specific variations or strains of $G$ described by the agent parameters as will be elaborated further in the sections that follow. 
Table 1. Family (Level 0) exposure cluster

\begin{tabular}{lcccc}
\hline Attitude, $\mathbf{G}$ & Resistance, $\mathbf{P}_{\mathbf{a}}$ & Resistance, $\mathbf{P}_{\mathbf{b}}$ & $\begin{array}{c}\text { Resulting } \\
\text { resistance }\end{array}$ & Outcome \\
\hline Type VS & Strong & Strong & Strong & Strongly suppressed \\
\hline Type S & Strong & Average & $\begin{array}{c}\text { Average to } \\
\text { Strong }\end{array}$ & Suppressed \\
\hline Type A & Average & Average & Average & Average propagation \\
\hline Type W & Weak & Weak & Weak & Strong propagation \\
\hline
\end{tabular}

Similar considerations can be applied to other groups, or exposure clusters, as discussed further in this work, with the outcome determined by the size and composition, that is, variation of immunity parameters in the group $i$, that can be described by the attitude type of the cluster with respect to the agent, $V_{i}(P, G)$.

\subsection{Cluster Hierarchy}

From the level of minimal (Level 0) clusters, a hierarchy of exposure clusters can be constructed based on the relation of proximity with respect to transmission of the infectious agent. Clusters at the Level $i$ immediately adjacent to each other are constructed based on their attitudes to the agent, with the resulting attitude of the next level cluster, with the attitude $V_{i+l}(P, G)$.

An important consideration that needs to be added in this analysis is variation in the infectious agent itself. For example, different Level 0 clusters can show different attitudes to different variations, or strains of $G$, therefore the resulting attitude is not a constant but rather a distribution with respect to infectious factors $G$. For example, a Level 1 cluster composed of family clusters A, B with attitudes: strong suppression of all strains; average suppression except specific strains $G_{k}$ would have the attitude of strong suppression for all strains of $G$ except $G_{k}$ and some resistance to $G_{k}$, i.e. a modal spectrum of resistance to $G$.

\subsection{Uniformity Threshold}

An assumption and hypothesis that is proposed is that there is a certain minimal size of a group at which the distributions of immunity parameters $P$ in the group are stabilized, meaning that the cluster contains sufficient sampling of the general population with respect to variation of immunity parameters with respect to the infectious agent to produce statistically stable response resulting from exposure to $G$. Statistical stability means that two different clusters of similar size produce same or similar probability distributions of the outcomes under the same parameters of exposure.

Consequently, clusters of the size beyond uniformity threshold can be characterized by a distribution over the infectious parameters alone:

$$
V_{U}(P, G)=V_{U}(G)
$$

describing a probability distribution, or spectrum of responses that can be expected from the cluster at the uniformity scale to an exposure to $G$. 


\section{Cluster Network Model}

With the model defined by a hierarchy of exposure clusters constructed as described in the preceding section we will consider propagation of an infectious agent $G$ in two scenarios: in the environment of natural immunity; and in a scenario with mass immunization with DIV-type vaccine(s). While detailed numerical analysis of scenarios will be a subject of another work, a number of qualitative considerations will be presented for both cases.

Of interest here will be two essential factors at the uniformity threshold level, as they would influence the development of the epidemiological scenario on a large populationwide scale. The first is the characteristic timeframe for emergence of stable strain $G_{S}$ on the uniformity scale, i.e. the time to produce a strain of $G$ to which clusters at uniformity scale will have attitude $V_{U}\left(G_{S}\right)$ compatible with unlimited propagation of $G$ in the population. The second factor is infectivity of $G$ at the point of achieving stability.

\subsection{Natural Immunity Scenario}

As mentioned earlier this scenario is characterized by significant variation of immunity parameters $P$ in the exposure clusters of the model.

As already mentioned in Sections 2.2 and 2.3, variation of natural immunity parameters in the exposure clusters from level 0 and above can have significant influence on higher level clusters and characteristic factors of interest. Based on the analysis in Section 2.2, clusters in the model at a given level $k$ can be seen as a network of nodes of different attitudes, from strongly resistant to conducting. In an additional dimension to that view, the attitude of the nodes can be expressed differently to different strains of $G$. For example, some nodes may suppress all strains while others would show different resistance based on characteristics of specific strains.

To produce a strain that is stable in a Level 1 cluster, it has to survive in a minimal number of Level 0 nodes. Let us consider for example, the case two Level 0 node cluster described in Section 2.3. After a short period past exposure it would be resistant to all strains (fully resistant clusters), as all strains would be suppressed in both nodes except the mode $G_{k}$, and the latter can be transmitted only within the node B that will eventually acquire immunity against this particular strain as well. It can be concluded then that an exposure to $G_{k}$ would convert this Level 1 cluster to a fully resistant one. It is clear that to attain stability even within the next, Level 1 cluster, a strain would have to possess some specific characteristics making it compatible with the immunity profiles of the nodes in the cluster, a factor that would impose constraints on the strains produced by random mutation (unless a "winning" strain is produced within certain window, the cluster may acquire full resistance and the game is lost for $G$ at least in this particular cluster).

Clearly, variation of natural immunity parameters can impose, via this mechanism, strong constraints on the development of new strains even for infectious agents with significant mutability, and provide additional, subject to confirmation by quantitative studies, population-wide protection effect against novel and emergent agents. Should a stable strain on the uniformity i.e. population-wide level emerge, it could take a 
considerable combination of attempts and random mutations to overcome this natural population-level immunity barrier. Then, the characteristic timeframe $\tau_{U}$ of achieving the stability can be expected to be a significant multiple (determined by the exact statistical model of the cluster network) of timeframes of producing a "significant mutation" (the term will be elaborated in the construction of a numerical model):

$$
\tau_{U}(G)=N \times \tau_{m}
$$

\subsection{Direct to Immunity Mass Vaccination Scenario}

With DIV immunization, an immunity response is triggered by production of viral proteins by body's own cells induced by mRNK message. In this case, the immune system of the immunized group of the population is programmed to counteract agents carrying certain protein signature.

An essential for this analysis difference with the case of natural immunity considered earlier can be illustrated by the following model. Consider the multi-dimensional vector describing the attitude of a population cluster to the agent $G$ in the coordinates of agent factors, i.e. characteristics describing the agent, in the direction of maximum resistance. In the natural population the vectors in different groups / clusters would point in different directions, as a result of natural variation in the immunity parameters in the population and composition of the clusters.

In the population immunized with DIV on the other hand, the parameters of maximum resistance would be predetermined by the proteins encoded in the vaccine's messenger, hence in this case, the vectors defining the direction of maximum resistance are pre-determined as well. Immune profiles, relevant to the agent, of the entire immunized population can be seen as aligned, or, using an analogy from the optics, polarized along the same resistance axis.

This observation is not unconditional and is based on the assumption that having been instructed to remember response to certain protein stimulus, the immune system would not remember also different alternative responses. Such "memory" is expensive and as experience with other rapidly evolving agents shows, may not be used in such cases by the immune system. These arguments provide ground for the model of polarized immune profile in the immunized population.

A critical factor for analysis of mass immunization is the rate, $\tau_{R}$, or probability of production of strains relative to the encoded "standard" profile, including those resistant ones (orthogonal, in the proposed model) to it, $\rho_{R}$. This factor has to be evaluated in a careful analysis based on specific parameters of the messenger and the agent that will not be attempted here.

The immediate observation based on the cluster model defined earlier, is that should a resistant strain $G_{R}$ be in fact, produced by a mutation of the agent, it would meet little resistance in propagating up the cluster hierarchy from the lowest to uniform level, in the immunized population. This conclusion follows immediately from the polarization pattern, with exposure clusters at all levels aligned in the same direction of group immunity profile, orthogonal to that of the resistant strain, that is defined by the vaccine's messenger. 
One has to conclude then that as a side effect of the immuno-polarization induced in this scenario, the population-wide protection effect produced by variation of immunity described in the preceding section would be effectively turned off. Where infectious agent had to produce strain resistant to multiple immune profiles simultaneously, it would be sufficient in this case to produce a single mutation resistant to the induced "standard" profile. The rate or probability of such mutation is fully defined by the characteristic parameter $\rho_{R}\left(\tau_{R}\right)$.

Another observation that can be made in this case is that not only, having been produced, the resistant strain would reach the uniformity scale almost unobstructed; but in the process of ascendance to the uniformity scale, the competition would proceed between resistant sub-strains of $G_{R}$ with selection by infectivity.

The conclusion for this scenario is that if a resistant strain can be produced by a mutating agent, it can be expected that the time to uniformity scale could be significantly shorter, while uniform infectivity higher than in the natural immunity case. By defining and imposing a "standard" population-wide immunity profile we also define the direction in which it can be broken through, almost immediately reaching population-level stability.

\subsection{Comparative Analysis}

The results in the preceding sections are sufficient for an intinial comparative analysis of the characteristic parameters in the considered scenarios of natural immunity and mass DIV vaccination. Indeed, for the timeframe of reaching the uniformity scale (i.e., population-level) stability, the ratio can be found from (2) and the analysis in Section 3.2. as:

$$
\frac{T_{n a t}}{T_{\text {div }}}=\frac{N_{\text {mod }} \times \tau_{m}}{\tau_{R}}=N_{\text {mod }} \frac{\tau_{m}}{\tau_{R}}
$$

where $T_{n a t}, T_{d i v}$, characteristic timeframes of reaching uniformity scale for the natural immunity and DIV scenarios, respectively; scenario parameters $\tau_{m}, \tau_{R}$ as discussed in Sections 3.1 and 3.2; and scale factor $N_{\text {mod }}$ obtained from the statistics of the cluster network model.

With a given cluster model, the scale factor is determined by the model and the parameters of immunity distribution in the population $X(p)$, and the characteristic timeframe will be defined by the ratio of the scenario factors, $\frac{\tau_{m}}{\tau_{R}}$. As mentioned earlier, the latter is determined by the specific factors of the immunity profile induced by the vaccines and needs to be analyzed carefully. Without attempting a detailed quantitative analysis that could be a subject of another study, a quick heuristic observation can be made here.

Suppose the target entity of the vaccine, such as specific proteins, that defines the immune profile of the maximum resistance in the agent parameter space $G$ is located in a mutable region of the agent's genome and thus itself is a subject to frequent mutation. It can be argued that in this case the scenario factor ratio in (3) can be close to 1, as the rate / probability of producing a strain resistant to a given profile, whether induced by the vaccine, or based on the parameters of distribution of immunity profiles in a given 
cluster should be approximately same, under the assumption of random isotropic mutation. In this case, the ratio of the timeframes in (3) would be determined by the scale factor $N_{m o d}$, confirming an essential role of immunity variation in population-level protection against novel strains. The natural immunity variation in the population in this case provides a type of magnification effect, increasing the timeframe of emergence of population-level stable strains, or slowing down the rate of production of such.

If, on the other hand, the target is located in a less mutable regions of the agent, the characteristic timeframe of production of a stable strain $\tau_{R}$ can be significantly greater than $\tau_{m}$ and the protection effect of the natural immunity scenario compared to mass vaccination will be reduced.

Based on the analysis in Section 3.2, a conjecture can be made on the infectivity of the produced strains at population-level stability. In the natural immunity scenario, the main selection factors for strains trying to achieve stability would be reaching the next cluster level (i.e. broader compatibility with the immunity profile distribution in the population); whereas in the case of mass DIV immunization, resistant strains would "float" to the uniformity scale with little resistance and the selection of the resulting strain is statistically likely to happen by infectivity. These arguments suggest that the resulting infectivity can be expected to be higher in the DIV scenario.

\subsection{DIV vs Natural Agent Vaccines}

To what extent considerations of the preceding section apply to the case of natural agent vaccines, including inactivated, live attenuated, dead, partial-vector and other types [3], is an interesting question that requires further analysis and discussion. From one perspective, these cases can be compared to a population immune response that can be triggered by a rapid onset of a natural epidemics. On the other hand, the model with imposed "standard" immunity profile interfering with the natural protection due to variation can be applicable as well, at least to some extent. This question merits further indepth investigation by the research community.

\section{Conclusions}

Vaccines, including novel direct-to-immunity types have shown strong promise in reduction of severe Covid-19 cases in clinical trials and early results of vaccination programs. However, as with many new developments, they may not be free of unintended and / or not anticipated longer-term effects. Cluster hierarchy approach proposed in this work points at a number of directions in the analysis of longer-term effects of mass immunization programs in scenarios with rapidly evolving infectious agents.

The first conclusion is that natural variation of immunity characteristics in the general population can provide non-trivial and possibly, significant protection against emergence of novel infections via imposing strong restrictions on emergent strains on the path to population-level stability. Natural variability of immunity in the population this provides additional defense against emerging infections. 
Secondly, mass vaccinations with DIV in rapidly evolving infectious environments introduced without careful analysis of long term effects and consequences may lead to, as an untended side effect, a weakening of that natural defense mechanism by inducing an alignment, or polarization of immunity profiles in the immunized population along predetermined direction, defining by the same token the mutation path to breaking through it. Long term exposure of the immunized population to infectious agent then may result in emergence of resistant strains with weakened population-level defense mechanism to slow down their ascendance to population-level stability. Further research and careful analysis of cluster network models may produce more confident quantitative results in this area.

A possible direction to reduce the impact of the immune-alignment effect would be to limit vaccination program to specific group, such as highly vulnerable or with extra exposure to the infection. This way, a significant pool of natural immunity variation in the general population can be retained with the effect of natural protection against emergent infectious strains discussed earlier in this work.

Another possible direction is to explore other mechanisms of stimulating general immunity not limited to specific agent(s) such as early-age exposure to certain agents that may trigger and stimulate development of such defense mechanisms [4,5].

Finally, the potential of technology-based methods in slowing the spread of agents should not be overlooked [6,7]. Slowing the transmission of infectious agent within and between exposure clusters can work as a "brake" in propagation of the agent to population-level stability (uniformity scale). If an emerging strain can be contained to a lower-level cluster, it would be likely to extinguish, preventing an emergence of another population-level variant.

It is expected that further analysis along the directions outlined in this work may yield answers to these and other questions on population-level immunity protection mechanisms against novel and evolving infections.

\section{References}

1. Pardi, N., Hogan, M.J., Porter F.W., Weissman, D., mRNA vaccines - a new era in vaccinology, Nature Reviews Drug Discovery, 17261 - 279 (2018).

2. The different types of Covid-19 vaccines, World Health Organization, https://www.who.int/news-room/feature-stories/detail/the-race-for-a-covid-19-vaccine-explained (January, 2021).

3. Types of vaccines, The Immunization Advisory Center, New Zealand https://www.immune.org.nz/vaccines/vaccine-development/types-vaccines (August, 2021).

4. Arts, R.J.W., Carvalho, A., La Rocca, C., Palma, C., Rodriguez, F. et al.: Immunometabolic pathways in BCG-induced trained immunity, Cell Reports, 17(10) 2562-2571 (2016).

5. Dolgikh, S.: Covid-19 vs. BCG universal immunization: statistical significance in the early phase of the pandemics, SciMedicine Journal, 3 55-66 (2021).

6. Kummitha, R.K.R: Smart technologies for fighting pandemics: the techno- and humandriven approaches in controlling the virus transmission, Government Information Quarterly, 37(3), 101481 (2020).

7. Dolgikh, S.: Smart-Covid: intelligent solutions for higher risk environments. HAL archivesouvertes (hal-02915459v2), (2020). 